\title{
Digestion, chez le lapin en croissance, d'une ration à taux élevé de constituants pariétaux : étude méthodologique pour le calcul de digestibilité apparente, par segment digestif
}

\author{
T. GIDENNE et C. PONCET \\ avec la collaboration technique de A. LAPANOUSE \\ I.N.R.A., Laboratoire de Recherches sur l'Elevage du Lapin \\ Centre de Toulouse, B.P. 27, F 31326 Castanet Tolosan Cedex \\ I.N.R.A., Laboratoire de la Digestion \\ C.R.Z.V. de Theix, F 63I22 Ceyrat
}

\begin{abstract}
Résumé
Une méthodologie nouvelle a été mise en œuvre, chez le lapin en croissance, pour estimer l'importance de la digestion dans différents segments du tube digestif. 27 lapins néo-zélandais blancs âgés de 8 semaines ont reçu une ration riche en glucides pariétaux ( 25 p. 100 de cellulose brute) et en lignine $(11$ p. 100$)$, et qui contenait un marqueur de la phase solide (l'oxyde de chrome mordancé). Les digesta ont été prélevés par abattage, toutes les 3 heures durant 24 heures, dans l'estomac, l'iléon terminal, le cæcum et le rectum. Les coefficients d'utilisation digestive apparents (CUDa), calculés par collecte totale des fèces, sont modestes pour la matière sèche (MS), l'énergie et les matières azotées (54 à 60 p. 100 tabl. 1). Les hémicelluloses (VAN SoEST) sont mieux digérées (CUDa $=40$ p. 100) que la cellulose (CUDa $=30$ p. 100), alors que 16 p. 100 des lignines semblent digérées.
\end{abstract}

L'association de la technique d'abattage et du marquage des aliments permet de déterminer la digestibilité et les temps moyens apparents de rétention aux différents sites de prélèvements et permet, de plus, d'estimer quantitativement la cæcotrophie. Ainsi, le CUDa de la MS est de 14,1 p. 100 avant le cæcum, contre 32,2 p. 100 au niveau du cæcum. Dans le cas de l'azote (tabl. 5), cette méthode ne semble pas aussi concluante : le CUDa est négatif au niveau iléal. L'hypothèse d'un transit différentiel entre les composants du régime est avancée. Les temps moyens apparents de rétention (tabl. 4) sont de 3 à 4 heures pour l'estomac, et de 4 à 6 heures pour le cæcum; ce qui est relativement plus faible que les valeurs de la littérature. La quantité de cæcotrophes produite quotidiennement atteint 15 p. 100 de l'ingéré total de matière sèche et 23 p. 100 de l'ingéré total de matières azotées.

Mots clés : Digestibilité, transit, constituants pariétaux, lapin en croissance, caecotrophie.

\section{Introduction}

La digestibilité de la ration chez le lapin est généralement étudiée en utilisant la méthode de collecte totale des fèces. Nous n'avons alors qu'une estimation glo- 
bale de la digestion. Peu d'auteurs ont tenté de calculer chez le lapin, la part de la digestion se déroulant dans les parties antérieures et postérieures (cæcum-côlon) du tube digestif. Seul Wolter et al. (1980) ont fait des mesures de digestibilités partielles au niveau de différents segments du tube digestif. Mais ils utilisaient un marqueur (l'oxyde de chrome non mordancé) peu fiable. De plus, les échantillons de contenus digestifs étaient obtenus après un abattage unique situé en dehors de la période de cacotrophie, et ils ne pouvaient donc rendre compte du flux digestif quotidien.

Ainsi, compte tenu du peu de travaux effectués auparavant sur ce sujet, avons-nous dû choisir et mettre en œuvre une méthodologie nouvelle permettant le calcul de bilans digestifs par compartiment.

Ce travail s'insère dans le cadre général de l'étude de l'utilisation digestive des parois végétales chez le lapin. C'est pourquoi nous appliquons notre méthode à l'étude d'un régime riche en parois et plus particulièrement à taux élevé en lignine VanSoest.

\section{Définition et choix méthodologique}

Les méthodes permettant d'estimer l'importance de la digestion au niveau de différents segments digestifs ont été mises au point initialement chez le ruminant. Deux problèmes majeurs doivent être résolus :

a) obtenir des échantillons représentatifs du contenu digestif transitant quotidiennement, au niveau de différents segments du tube digestif, pour l'étude de leur composition ;

b) quantifier le flux digestif au niveau des sites considérés.

Le premier problème est généralement résolu chez le ruminant par la pose de canules (simples ou réentrantes) au niveau du rumen, ou du duodenum...

Chez le lapin, la digestion microbienne a lieu essentiellement au niveau de l'ensemble cæcum-côlon. La pose de fistule cæcale est réalisable chez le lapin (Yoshida \& Kandatsu, 1967 ; Le Bars et al., 1971 ; Susmel \& Lanari, 1976). Par contre, la création de fistule iléale est plus difficile (KAMETAKA, 1967; Leng \& HorNICKE, 1975). De plus, il est peu envisageable de placer sur le même animal plus de deux canules, donc de prélever à plus de 2 points du tube digestif. La composition du contenu digestif peut varier sensiblement au cours du nycthémère, principalement en fonction de la pratique de la cacotrophie (Catala, 1976; Demaux et al., 1980) ; aussi serait-il nécessaire de réaliser des prélèvements fréquents, qui ne doivent pas interférer avec le fonctionnement normal du tube digestif. Nous avons donc effectué des prélèvements de contenus digestifs par la méthode des abattages, malgré la variabilité interanimal qui en découle, car ceci permet de résoudre les deux problèmes précédents : prélèvement fréquent, et en autant de points que nécessaire.

Les abattages ont lieu toutes les 3 heures durant 24 heures. Nous prélevons à 4 niveaux du tube digestif : 
- l'estomac : pour une estimation quantitative de la cæcotrophie et une mesure de son influence sur les flux digestifs à ce niveau ;

- iléon terminal : à ce niveau, la digestion enzymatique prend fin, et nous pouvons déterminer la composition des digesta entrant dans le cæcum;

- cæcum : principal lieu de la digestion microbienne chez le lapin ;

- rectum : ce prélèvement permet de situer au cours de la journée la formation de cæcotrophes (crottes molles) et de crottes dures, et permet bien sûr de déterminer le bilan digestif global en comparaison avec le coefficient d'utilisation digestive apparente (CUDa), calculé après récolte totale des fèces.

Le second problème est de connaître la quantité de contenu digestif ayant transité pendant une journée sans être obligé de collecter la totalité du flux. Une mesure de flux peut s'effectuer par débitmétrie, dans le cas de flux liquide (sang); mais dans le cas de flux digestif, le marquage des digestas est la méthode la plus couramment employée.

Les données actuelles sur la mesure de flux chez le ruminant sont nombreuses et très variables selon la méthode utilisée. FAICHNEY (1980) propose une méthode à double marquage ; un marqueur de la phase solide et un marqueur de la phase liquide. Ceci semble nécessaire chez le ruminant, dans la mesure où le rumen est un compartiment de mélange, avec 2 phases distinctes ne transitant pas de la même façon, et dont il est difficile d'obtenir un échantillon représentatif par fistulation.

Chez le lapin, l'estomac est un compartiment au contenu hétérogène, mais il n'est pas le lieu d'une digestion intense, à l'opposé de l'intestin grêle ou du cacum. Ces derniers par contre, ont un contenu homogène (Yoshihara, Kandatsu, 1960, cité par LAPLACE, 1978) et ne sont pas assimilables à un compartiment de mélange. De plus, la méthode des abattages nous permet de prélever en totalité le contenu du segment, et donc d'obtenir un échantillon représentatif.

Dans notre cas, il semble donc suffisant d'utiliser un marqueur de la phase solide. Actuellement, plusieurs études ont montré la validité du marquage avec les terres rares pour l'étude du transit chez le ruminant. Le cerium a été utlisé, chez le lapin, par LebAs \& LAPlace (1977), mais sans que soit montrée la validité (étude comparative) de ce marquage pour cette espèce. Depuis ces travaux, sont apparues les techniques de mordançage, testées chez le lapin par UDEN et al. (1980). Les auteurs montrent que la préparation du complexe fibre-chrome aboutit à des liaisons résistantes aux agents de la digestion : 98 p. 100 du marqueur restent fixés aux fibres après digestion. En plus des qualités principales demandées à tout marqueur de transit (Котв \& Luckey, 1972), le chrome a l'avantage d'être facilement dosable dans les contenus digestifs.

Le mode d'administration du marqueur doit être choisi pour obtenir un état stable dans les digesta au cours de la journée. Les techniques d'infusion à l'aide de fistules du rumen sont alors utiles chez le polygastrique pour atteindre la stabilité, étant donnés les problèmes d'homogénéisation du marqueur avec la ration.

Dans notre étude, le foin de luzerne mordancé au chrome est homogénéisé avec le reste de la ration, puis l'ensemble est granulé. Les lapins ne peuvent donc trier les fibres marquées parmi les autres composants de la ration. 


\section{Matériel et méthodes}

\section{A. Matériel animal-alimentation}

Vingt-sept lapins néozélandais blancs, âgés de 8 semaines ont été élevés en cage individuelle, dont 10 en cage à digestibilité. Durant une période de 10 jours, ils reçoivent, à volonté, un aliment granulé. Dans celui-ci, du foin de luzerne mordancé avec le chrome, selon la technique décrite par UDEN (1978), a été incorporé à raison de 5 p. 100 de la matière sèche (MS). En outre, cette ration contient : 74,4 p. 100 de luzerne déshydratée $-13,3$ p. 100 de pellicules de colza - 3,8 p. 100 de tourteau de soja - 2,6 p. 100 d'huile de colza et 0,9 p. 100 de composé vitaminique et minéral. La composition chimique figure au tableau 1.

\section{TABLEAU 1}

Analyse de l'aliment et CUDa.

Experimental diet composition and apparent digestibility.

\begin{tabular}{|c|c|c|c|}
\hline \multirow{2}{*}{$\begin{array}{l}\text { Composants } \\
\text { Ingredients }\end{array}$} & \multirow{2}{*}{ Résultats d'analyse } & \multicolumn{2}{|c|}{ CUDa p. 100} \\
\hline & & $\overline{\mathbf{x}}$ & $\sigma$ \\
\hline Matière sèche - Dry matter & 91,2 & 54,5 & 1,8 \\
\hline $\begin{array}{l}\text { Energie brute }(\mathrm{kcal} / \mathrm{kg} \text { MS }) \ldots \ldots \ldots \\
\text { Crude energy }(D M)\end{array}$ & 4516 & 52,4 & 3,9 \\
\hline $\begin{array}{l}\text { Matière organique (p. } 100 \text { MS) } \ldots \ldots \ldots \ldots \ldots \\
\text { Organic matter }\end{array}$ & 80,6 & 55,3 & 1,9 \\
\hline $\begin{array}{l}\text { Protéines brutes (p. } 100 \mathrm{MS} \text { ) } \ldots \ldots \ldots \ldots \ldots \ldots \\
\text { Crude protein }\end{array}$ & 14,0 & 60,5 & 1,9 \\
\hline Cellulose «Weende»(p. $100 \mathrm{MS})$ & 25,4 & 21,7 & 2,9 \\
\hline NDF (p. 100 MS) $\ldots \ldots \ldots \ldots$ & 47,8 & 30,5 & 2,3 \\
\hline Hemicellulose VAN SoEst (p. $100 \mathrm{MS}$ ) & 14,0 & 40,5 & 0,9 \\
\hline Cellulose VAN SOEST (p. 100 MS) & 22,8 & 32,2 & 0,5 \\
\hline Lignine (p. 100 MS) & 11,2 & 15,9 & 5,7 \\
\hline
\end{tabular}

\section{B. Abattages - Prélèvements et dosages}

Toutes les 3 heures, 3 animaux sont abattus, pendant 24 heures à partir de 9 heures du matin. Immédiatement après dislocation cervicale et saignée, le tube digestif complet est isolé. Nous prélevons le contenu des 50 derniers centimètres de l'iléon, des 20 derniers centimètres du rectum, et les contenus totaux de l'estomac et du cæcum.

A chaque heure de prélèvement, les digesta issus de chaque animal sont groupés par site digestif, après contrôle des poids frais et du pH (GidenNE \& LeBAs, 1984). 
Les dosages sont effectués sur les produits secs $\left(24 \mathrm{~h}\right.$ à $\left.103^{\circ} \mathrm{C}\right)$. Après minéralisation à l'acide nitroperchlorique, l'oxyde de chrome est dosé au Technicon avec la diphenylcarbazide (méthode de Mathieson J., 1970). Nous avons déterminé les teneurs en protéines brutes (azote Kjeldhal $\times 6,25$ ) en énergie (calorimètre adiabatique Gallenkamp) ainsi qu'en cellulose brute (méthode de Weende) et en constituants pariétaux dosés selon la méthode de VAN SoEST \& Wine (1967).

\section{Mesures de digestibilité}

Après 10 jours d'accoutumance, la digestibilité des différents composants de la ration est évaluée par collecte totale journalière des fèces, durant 3 jours. Ceci nous permet en outre d'estimer le taux de récupération du marqueur. Les bilans digestifs par compartiment ont été calculés à l'aide du marqueur, pour la matière sèche et la matière azotée selon la méthode suivante : la quantité de matière sèche (QMS exprimée en g) ayant transité au niveau d'un organe est :

$$
\mathrm{QMS}=\frac{100 \times \mathrm{Q}}{\mathrm{C}}
$$

avec $Q=$ quantité de marqueur ayant transité en $24 \mathrm{~h}=$ quantité ingérée ( $\mathrm{g}$ ) en $24 \mathrm{~h}$ (pour un taux de récupération de $100 \mathrm{p}$. 100).

$\mathrm{C}=$ concentration moyenne au cours de $24 \mathrm{~h}$ de ce marqueur dans l'organe considéré exprimé en g pour $100 \mathrm{~g}$ MS.

Le calcul du flux de matière azotée est obtenu en multipliant la valeur du flux de MS (QMS) par la teneur moyenne en azote du contenu. Le principe de ce calcul peut à l'évidence être appliqué à tout autre composant de la matière sèche.

\section{Résultats et discussion}

\section{A. Digestibilité globale de la ration}

Les valeurs des coefficients d'utilisation digestive apparente (CUDa) (tabl. 1) sont corrigées en fonction des 5 p. 100 de foin de luzerne mordancé au chrome, totalement indigestible. Les calculs ne portent que sur 3 jours de collecte et non sur 2 périodes de 4 jours (Colin \& Lebas, 1976).

Toutefois, nous remarquons des valeurs assez faibles pour le CUDa de la MS, de l'énergie et de l'azote (52-60 p. 100), alors que 16 p. 100 de la lignine semblent digérés. La teneur élevée en constituants pariétaux de la ration pourrait expliquer en partie ces faibles CUDa : un apport élevé de «fibres » abrège le temps de transit et serait susceptible de réduire la digestibilité des matières azotées. Mais, LeBAs (1975) et LebAS et al. (1982) n'obtiennent pas de baisse sensible du CUDa des protéines avec un apport croissant de cellulose. L'effet du taux de constituants pariétaux ne peut donc être retenu. Par contre, la composition des parois végétales peut aussi intervenir. En effet, notre ration possède une teneur élevée en lignine $\left(\begin{array}{lll}11 & \mathrm{p} . & 100\end{array}\right)$ connue pour son effet inhibiteur sur les fermentations du rumen (AKIN, 1982). Ainsi 
les matières non dégradées arrivant au cæecum ne seraient en fait que partiellement dégradées par la flore, entraînant des CUDa modestes. Randall (1977) signale aussi sur des lapins adultes une baisse de la digestibilité de la ration quand le taux de lignine s'élève.

Nos résultats indiquent une digestibilité des hemicelluloses «Van Soest» (40 p. 100) supérieure à celle de la cellulose «Van Soest» $(30$ p. 100). D'autres auteurs ont obtenu chez le lapin, ce même type de résultats (UDEN, 1978 ; RANDALL, 1977). Le lapin semble donc mieux utiliser les hémicelluloses «Van Soest» que la cellulose «Van Soest », alors que les résultats obtenus chez les polygastriques indiquent fréquemment l'inverse.

L'utilisation digestive apparente de la lignine n'est pas négligeable, si l'on s'en tient au calcul du CUDa; mais nous enregistrons des variations inter-individuelles importantes : le CUDa de la lignine (Van Soest) varie de 8 à 25 p. 100 selon l'animal. Ceci montre à nouveau que la lignine dosée par la méthode de Van Soest ne peut être chez le lapin un marqueur fiable. Une méthode de dosage plus spécifique isolant un résidu lignine réellement indigestible permettrait sans doute son utilisation en tant aue marqueur.

\section{B. Taux de récupération du chrome et évolution nycthémérale de sa concentration dans le tractus digestif}

Pour vérifier la non-absorption du marqueur au cours du transit, nous avons calculé son taux de récupération dans les fèces, sur les 10 animaux èn cage à digestibilité. La valeur moyenne obtenue est de 102,8 p. $100 \pm 7,5$ p. 100 . UDEN et al. (1980) obtiennent aussi (sur 2 lapins) une valeur supérieure à 100 p. 100 (104,9 p. 100 $\pm 4,2$ p. 100). Le marqueur n'est donc pas absorbé au cours du transit.. Une récolte de fèces trop courte ( 3 jours seulement) et la précision de la méthode de dosage sont les principales causes d'un écart-type assez élevé et d'une valeur moyenne supérieure à 100 p. 100 .

Le chrome mordancé est un marqueur de la phase solide (et plus particulièrement de la fraction pariétale). Si dans un même segment digestif, la digestion demeure constante au cours du temps, alors l'évolution sur 24 heures de la concentration en marqueur (en p. 100 de la MS) devrait être exprimée par une droite horizontale. La figure 1 présente cette évolution aux 4 sites de prélèvement.

Il est impossible de tester la linéarité de ces courbes ainsi que la nullité de leur pente (horizontalité) car nous n'avons qu'une valeur par segment et par heure de prélèvement. Nous pouvons seulement nous référer aux valeurs moyennes des concentrations en chrome (tabl. 2) et à leurs coefficients de variation '(CV). Ces derniers sont proches des valeurs classiquement rencontrées lors d'essais semblables chez le ruminant (Ellis \& Huston, 1968 ; PonCET, 1985, données non publiées) sauf pour la valeur moyenne au niveau iléal dont le CV est de 16,6 p. 100.

Au cours du transit, la digestion de la matière sèche devirait entraîner une élévation de la teneur moyenne en chrome. Cette teneur s'élève effectivement de l'iléon au rectum (tabl. 2), mais au niveau stomacal elle est supérieure à celle de l'iléon. Pour mieux discuter ultérieurement de cette répartition du marqueur et ses variations de concentration (fig. 1), nous présentons à la figure 2, l'évolution du taux de matière sèche des contenus digestifs, et à la figure 3 une chronologie de la pratique de la cacotrophie de nos animaux. Nous constatons une relative stabilité des teneurs en 
matière sèche, sauf au niveau rectal, où des variations sont enregistrées en relation avec la dualité de l'excrétion fécale. Les teneurs en matières azotées totales (MAT) évoluent de manière significative $(P<0,01)$, au cours du nycthémère, quelle que soit la zone digestive considérée (fig. 4). La pratique de la cacotrophie a lieu dans la matinée (entre $6 \mathrm{~h}$ et $12 \mathrm{~h}$ ), et aussi dans une faible mesure, au cours de la nuit (entre 3 et $6 \mathrm{~h}$ du matin). Cette seconde phase de cæcotrophie a déjà été signalée par LAPLACE (1978), chez le lapereau.

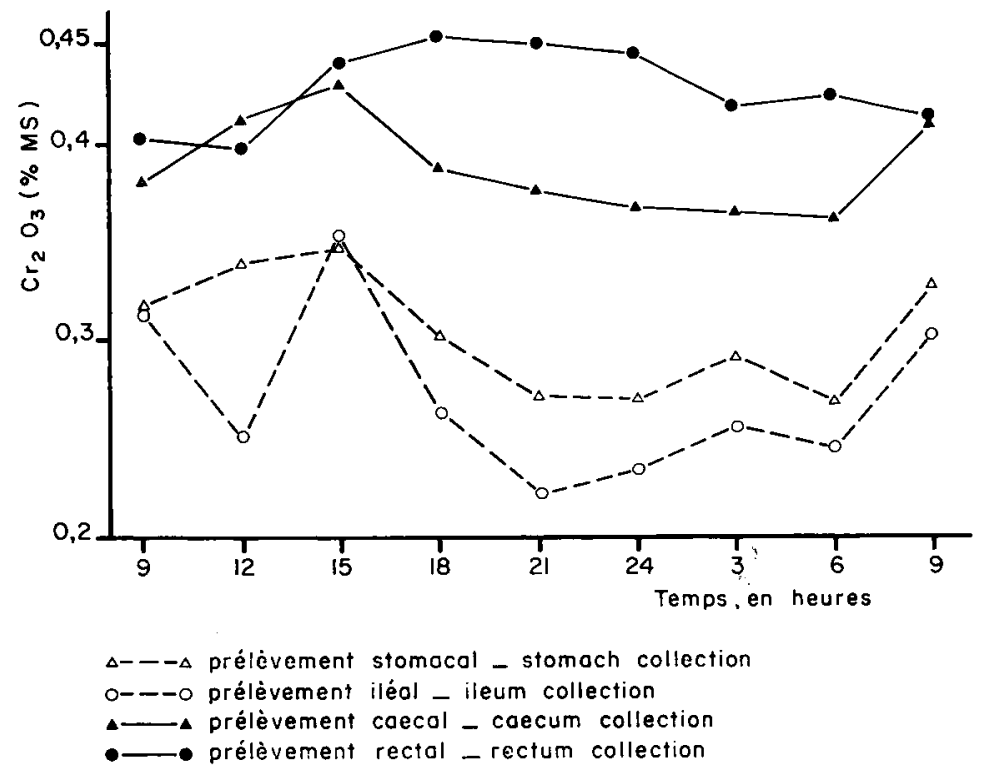

FIG. 1

Evolution nycthémèrale de la teneur en oxyde de chrome des digesta, en fonction du lieu de prélèvement.

Circadian changes in the chromium-oxide content of digesta according to collection site.

\section{Tableau 2}

Concentration en oxyde de chrome des digesta : moyennes de 9 observations/24 h ( $\mathrm{mg} / \mathrm{g} \mathrm{MS}$ ).

Concentration of chromium-oxide in digesta : mean of 9 observations in 24 hours ( $\mathrm{mg} / \mathrm{g} \mathrm{DM}$ ).

\begin{tabular}{|c|c|c|c|c|}
\hline Organe & $\begin{array}{l}\text { Estomac } \\
\text { Stomach }\end{array}$ & $\begin{array}{l}\text { Iléon } \\
\text { Ileum }\end{array}$ & Cæcum & Rectum \\
\hline Moyenne - Mean & 3,04 & 2,69 & 3,88 & 4,30 \\
\hline Ecart-type - Standard deviation & 0,32 & 0,45 & 0,25 & 0,19 \\
\hline $\begin{array}{l}\text { Coefficient de variation p. } 100 \ldots \ldots \ldots \ldots \ldots \\
\text { Variation coefficient }\end{array}$ & 10,4 & 16,6 & 9,2 & 4,4 \\
\hline
\end{tabular}




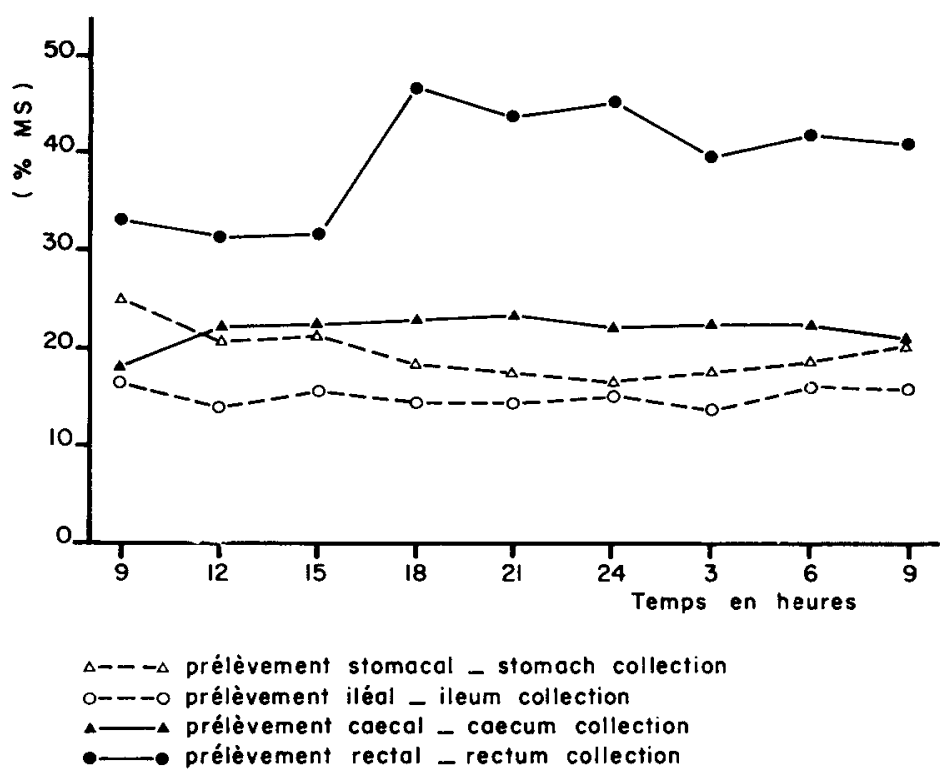

FIG. 2

Evolution nycthémèrale de la teneur en matière sèche des digesta, en fonction du lieu de prélèvement.

Circadian changes in the dry matter content of digesta according to collection site.

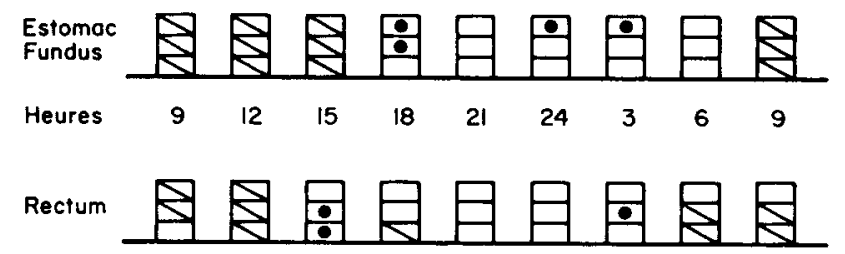

absence de coecotrophe no caecotrophe

- présence de quelques coecotrophes (estomac) ou de crottes intermédioires (rectum)

some coecotrophe (stomoch), or intermediate feces (rectum)

$\square$ présence de coecotrophes presence of coecotrophe

NB : une cose équivout $\dot{a}$ un lopin each square is equivalent to one rabbit

FIG. 3

Présence de caecotrophes dans le tube digestif des animaux expérimentaux au cours de la journée.

Presence of caecotrophes in the stomach and rectum of rabbits. 


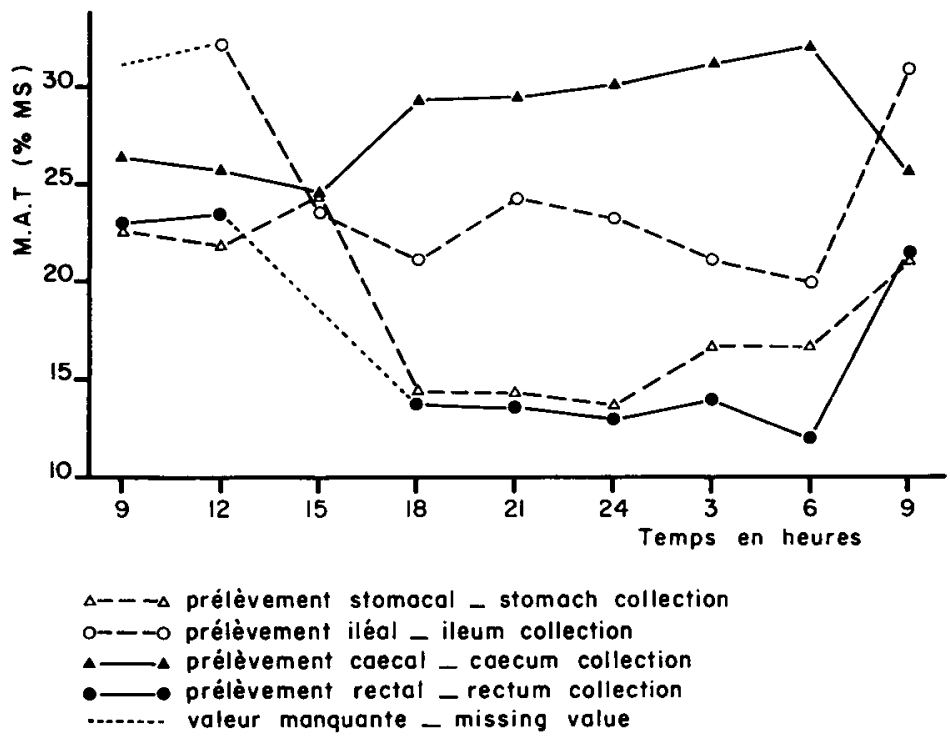

FIG. 4

Evolution nycthemérale de la teneur en matières azotées totales des digesta, en fonction du site de prélèvement.

Circadian changes in the total crude protein content of digesta according to collection site.

TABleau 3

Evolution nycthemérale du contenu stomacal.

Circadian changes in gastric contents.

\begin{tabular}{|c|c|c|c|c|c|c|c|c|c|}
\hline $\begin{array}{c}\text { Heures } \\
\text { Hours }\end{array}$ & 9 & 12 & 15 & 18 & 21 & 24 & 3 & 6 & 9 \\
\hline $\begin{array}{l}\text { Contenu sec }(\mathrm{g}) \text { total } \ldots \ldots \ldots \\
\text { Dry content }\end{array}$ & 29,8 & 23,3 & 22,8 & 17,4 & 15,0 & 16,0 & 18,2 & 16,6 & 22,0 \\
\hline $\begin{array}{l}\text { Contenu total en chrome }(\mathrm{dg}) \\
\text { Total content of chrome }\end{array}$ & 0,95 & 0,79 & 0,79 & 0,53 & 0,41 & 0,43 & 0,53 & 0,44 & 0,72 \\
\hline $\begin{array}{l}\text { Contenu sec }(\mathrm{g}) \text { en cæcotrophes } \\
\text { Dry content of caecotrophes }\end{array}$ & 11,8 & 12,9 & 14,1 & 4,9 & 0,7 & 0,4 & 3,5 & 0,0 & 7,6 \\
\hline $\begin{array}{l}\text { Contenu sec }(\mathrm{g}) \text { en aliment } \ldots \\
\text { Dry content of food }\end{array}$ & 18,0 & 10,4 & 8,7 & 12,5 & 14,3 & 15,6 & 14,7 & 16,6 & 14,4 \\
\hline
\end{tabular}




\section{Estimation quantitative de la cacotrophie}

Nous avons prélevé, par abattage, la totalité du contenu stomacal ; nous connaissons ainsi son contenı en chrome aux différentes heures de prélèvements (tabl. 3). De même, nous connaissons la teneur en chrome des cæecotrophes (fig. 1, courbe rectum, valeur à $12 \mathrm{~h}$ : le rectum des 3 lapins contenait des cæcotrophes), et celle de l'aliment. Nous pouvons en déduire la quantité de chrome provenant de l'aliment et des cæcotrophes, et par conséquent la quantité d'aliment et de cæcotrophes présents dans l'estomac aux différentes heures de prélèvements. Soit :

$\mathrm{QE}=$ contenu sec total de l'estomac ;

$\mathrm{QA}=$ contenu stomacal sec, cacotrophes exceptés (fraction «alimentaire» du contenu stomacal);

$\mathrm{QC}=$ contenu sec, en cacotrophes, de l'estomac.

$\mathrm{E}=$ concentration en chrome du contenu stomacal total;

A = concentration en chrome de la fraction «alimentaire» du contenu stomacal ;

$\mathrm{C}=$ concentration en chrome des cxecotrophes (fig. 1, courbe rectum, valeur à $12 \mathrm{~h}$ ).

Nous avons :

(1) $\mathrm{QE}=\mathrm{QC}+\mathrm{QA} \Leftrightarrow \mathrm{QA}=\mathrm{QE}-\mathrm{QC}$

(2) $\mathrm{QE} \times \mathrm{E}=(\mathrm{QA} \times \mathrm{A})+(\mathrm{QC} \times \mathrm{C})$

d'où, en remplaçant la valeur de $\mathrm{QA}$ dans (2) :

$\mathrm{QE} \times \mathrm{E}=((\mathrm{QE}-\mathrm{QC}) \times \mathrm{A})+(\mathrm{QC} \times \mathrm{C})$

ainsi $(\mathrm{QE} \times \mathrm{E})-(\mathrm{QE} \times \mathrm{A})=\mathrm{QC}(\mathrm{C}-\mathrm{A})$ d'où $\mathrm{QC}=\frac{\mathrm{QE}(\mathrm{E}-\mathrm{A})}{\mathrm{C}-\mathrm{A}}$

De plus, à $6 \mathrm{~h}$, la valeur de $\mathrm{E}$ est minimale, la part du chrome provenant des cæcotrophes est donc minimum. L'estomac ne contient alors aucun cæcotrophe visible lors du prélèvement, mais quelques résidus provenant de cacotrophes ingérés la veille (ou le matin même à $3 \mathrm{~h}$ ) peuvent encore être présents. Aussi, nous utiliserons la valeur de $\mathrm{E}$ à $6 \mathrm{~h}$ en tant que valeur de $\mathrm{A}$ afin de tenir compte de ces éventuels résidus, pour calculer la quantité de cæcotrophes nouvellement ingérée au cours de la journée. Le calcul de la quantité de cæcotrophes présente dans l'estomac n'est effectué quavec 3 animaux par site digestif. Aussi, est-il nécessaire d'être prudent vis-à-vis des valeurs absolues présentées au tableau 3 (4* ligne). Par contre, la comparaison de ces valeurs nous montre une chute brutale du contenu en cæcotrophes de l'estomac au cours de l'après-midi (entre $15 \mathrm{~h}$ et $18 \mathrm{~h}$ pour nos essais). L'évacuation des cæcotrophes riches en azote $(23,5$ p. 100 de MAT) se répercute sur la teneur stomacale en MAT qui diminue de 22 p. 100 entre 15 h et 18 h (fig. 3). En accord avec HoRnicke (1980), l'évolution du contenu en cæcotrophes de l'estomac indique un stockage en début de journée, puis une évacuation assez rapide quelques heures plus tard. Il semble donc que chez le lapin, l'estomac ne soit pas qu'un simple organe de stockage mais qu'il joue un rôle particulier dans le tri et l'évacuation de son contenu. 
Nous pouvons donc estimer la quantité moyenne totale de cæcotrophes ingérés pendant $24 \mathrm{~h}$ grâce au rapport des concentrations moyennes (sur $24 \mathrm{~h}$ ) en chrome des cæcotrophes $(C=4,01 \mathrm{mg} / \mathrm{g} \mathrm{MS})$, de l'estomac $(E=3,01)$, et du contenu stomacal en aliment $(\mathrm{A}=$ valeur de $\mathrm{E}$ à $6 \mathrm{~h}=2,68)$. Ainsi, nous avons :

$\mathrm{E}=(\mathrm{x} \cdot \mathrm{A})+(\mathrm{y} \cdot \mathrm{C})$ et $\mathrm{x}+\mathrm{y}=1$ avec

$\mathbf{x}=$ proportion de chrome apportée par l'aliment ;

$y=$ proportion de chrome apportée par les cæcotrophes.

D'où $y=\frac{3,01-2,68}{4,01-2,68}=0,248$ et $x=0,752$

L'aliment apporte donc 75,2 p. $100 \mathrm{du}$ chrome total ingéré ; or l'ingéré quotidien de matière sèche est de $125,6 \mathrm{~g}$ à $0,215 \mathrm{p}$. $100 \mathrm{de}$ chrome soit $270,3 \mathrm{mg}$. Le chrome apporté par les cæcotrophes représente 33 p. 100 du chrome apporté par l'aliment, soit $270,3 \times 0,330=89,1 \mathrm{mg}$.

La quantité moyenne de cæcotrophes (QC) ingérée en 24 h est donc :

$$
\mathrm{QC}=\frac{89,1}{\mathrm{C}}=22,2 \mathrm{~g} \text { de } \mathrm{MS} \text {. }
$$

QC représente donc 15 p. 100 de l'ingéré total de matière sèche (aliment plus cæcotrophes). Sachant que la teneur en MAT des cæcotrophes est de 23,5 p. 100 (valeur à $12 \mathrm{~h}$ de la courbe rectum : fig. 3), leur apport azoté est donc de $5,2 \mathrm{~g} / 24 \mathrm{~h}$, soit 23 p. 100 de l'ingéré azoté total. Hornicke (1980) estime que la production de cæcotrophes varie entre 5 et 18 p. 100 de la matière sèche ingérée, et qu'elle peut atteindre 30 p. 100 de l'ingéré total de matières azotées.

\section{Temps de rétention apparent et flux digestifs}

La méthode des abattages permet de connaître la valeur moyenne du contenu sec stomacal et cæcal, et nous pouvons alors calculer le temps de rétention apparent (TRa). Il est défini par Minson (1966) comme le temps moyen pendant lequel séjourne un composé chimique dans le segment considéré : TRa = quantité de composé dans le contenu/quantité entrant chaque heure, dans le compartiment digestif. Les valeurs du TRa pour plusieurs composants de la ration sont calculées pour l'estomac et le cæcum (tabl. 4). Pour ce calcul, ainsi que pour le calcul des flux digestifs, il est nécessaire de connaître la quantité moyenne de cæcotrophes ingérée chaque jour. En effet, chez le lapin l'ingéré journalier est égal à l'ingéré d'aliment plus l'ingéré de cæcotrophes (estimé au paragraphe précédant à $22 \mathrm{~g}$ de MS pour nos essais); il en est de même pour le flux de digesta qui peut se décomposer en un flux d'origine alimentaire et en un flux provenant de l'ingestion des crottes molles.

Précisons qu'au niveau du cæcum, la quantité entrante d'un composé équivaut au flux iléal de ce composé, sauf dans le cas de la cellulose brute, où nous avons remplacé le flux iléal par l'ingéré total de cellulose brute, en soutenant l'hypothèse que celle-ci n'est que très peu digérée avant l'entrée dans le cæcum. 
Les valeurs des TRa au niveau du cæcum (4 à 6 heures) sont toujours supérieures à celles calculées au niveau stomacal ( 3 à 4 heures), sauf dans le cas de la cellulose brute. LeBAS (1979) estime que le temps de séjour moyen des aliments est de 4 à $6 \mathrm{~h}$ dans l'estomac, et de 6 à $9 \mathrm{~h}$ dans le cæcum. Nos valeurs sont inférieures; mais le régime riche en parois végétales $(\mathrm{NDF}=47,8$ p. 100) que nous employons peut l'expliquer. En effet, LeBAS \& LAPLACE (1977) observent une réduction du temps de rétention digestif total quand le taux de cellulose du régime augmente. Au niveau stomacal le TRa de l'azote $(4,2 \mathrm{~h})$ est supérieur à celui de la MS $(3,2 \mathrm{~h})$; ceci appuie l'hypothèse d'une rétention préférentielle des cæcotrophes (riches en azote). Le même phénomène, moins marqué, existe aussi au niveau cæcal $(4,8 v s 4,2)$. Dans ce cas, nous pouvons l'attribuer à l'activité du colon proximal qui refoule les fines particules et les solutés vers le cæcum. Minson (1966) obtient des résultats similaires au niveau du réticulo-rumen de mouton. Mais dans le cas des polygastriques, le phénomène est bien sûr différent. Minson (1966) pense que les bactéries qui métabolisent l'azote resteraient «attachées » à la paroi, d'où un temps de rétention plus élevé des matières azotées.

\section{Tableau 4}

Temps de rétention apparents (TRa) et flux digestifs (FD), en fonction du site de prélèvement.

Apparent retention time and digesta flow according to collection sites.

\begin{tabular}{|c|c|c|c|c|c|c|c|}
\hline \multirow[b]{2}{*}{ Organes } & \multicolumn{4}{|c|}{ TRa (heure) - hr } & \multicolumn{3}{|c|}{ FD $(g / 24 h)$} \\
\hline & $\begin{array}{l}\text { MS } \\
D M\end{array}$ & $\begin{array}{c}\text { MAT } \\
C P\end{array}$ & $\begin{array}{l}\mathrm{CB} \\
C F\end{array}$ & $\mathrm{Cr}_{2} \mathrm{O}_{4}$ & MS & MAT & C.B \\
\hline Estomac - Stomach & 3,2 & 4,2 & 4,0 & 4,0 & 119,0 & 24,1 & 36,7 \\
\hline Iléon - Ileum ... & - & 一 & - & - & 130,8 & 32,2 & $\mathrm{NC}$ \\
\hline Cæcum & 4,2 & 4,8 & 3,4 & 5,8 & 92,4 & 26,1 & 21,69 \\
\hline Rectum $\ldots \ldots \ldots$ & 一 & - & - & 一 & 83,3 & $\mathrm{NC}$ & $\mathrm{NC}$ \\
\hline
\end{tabular}

NC : Non calculable dans nos conditions d'expérience - Non calculable in our experimental conditions.

Les valeurs des flux digestifs, calculées à l'aide de notre marqueur, sont présentées au tableau 4. Aux niveaux rectal et iléal, les quantités de digesta prélevées étaient faibles (quelques grammes), et ne permettaient pas le dosage de la cellulose, ni celui des MAT (sauf dans l'iléon). Par conséquent, nous n'avons pas pu calculer les flux digestifs correspondants. Etant donné que la concentration en chrome de l'iléon est inférieure à celle de l'estomac, le flux stomacal de matière sèche (et de matière azotée) est donc inférieur à celui de l'iléon. Notre méthode de marquage ne semble donc pas utilisable dans le cas d'un compartiment de mélange (contenu hétérogène) tel que l'estomac. Pourtant dans le cas du flux stomacal quotidien de cellulose brute, notre estimation $(36,7 \mathrm{~g})$ semble correcte, puisque elle est très proche de l'ingéré total moyen de cellulose brute qui est de $37,1 \mathrm{~g}$ (soit $31,9 \mathrm{~g}$ de cellulose brute venant de la ration $+5,2 \mathrm{~g}$ venant des cæcotrophes). De plus, il en est de même dans le cas du flux stomacal des matières azotées : $24,1 \mathrm{~g} / 24 \mathrm{~h}$ contre $22,8 \mathrm{~g} / 24 \mathrm{~h}$ d'ingéré azoté total journalier. 


\section{E. Mesures de digestibilité selon le segment du tube digestif}

Le calcul des flux digestifs nous permet d'estimer les CUDa par compartiment digestif (tabl. 5), sauf dans les cas où le calcul des flux n'a pu être fait (paragraphe précédent).

Nous constatons que 40 p. 100 de la matière sèche ingérée disparaissent apparemment dans l'ensemble cæcum-côlon, soit près de 75 p. 100 de la matière sèche digestible. Ceci souligne l'importance de ce compartiment digestif et de sa flore en présence d'une ration riche en constituants pariétaux. La digestibilité totale de la MS est alors de 53,9 p. 100, soit une sous-estimation de moins de 1 p. 100 par rapport au calcul du CUDa par collecte totale des fèces.

Cependant, les valeurs négatives que nous obtenons dans le cas du CUDa des protéines brutes avant le cæcum, montrent l'imperfection de ce marqueur. Wolter et al. (1980), utilisant l'oxyde de chrome non mordancé, obtiennent avec un régime contenant 25 p. 100 de cellulose VaN SoEst des CUDa similaires aux nôtres dans le cas de la matière sèche. Dans le cas des matières azotées, cet auteur obtient un CUDa cumulé de -50 p. 100 avant le cæcum, de -100 p. 100 dans le cæcum; et de +57 p. 100 au niveau du colon distal.

Dans notre cas, la valeur cumulée au niveau cæcal n'est que de -20 p. 100 , mais nous n'avons pas pu poursuivre ce calcul jusqu'au niveau du côlon distal, faute de matériel pour analyse. Nous estimons à $6,1 \mathrm{~g}$ la quantité de MAT disparaissant dans le cæcum (CUDa $=+36,6$ p. 100) soit plus de la moitié de l'azote digestible. Ceci soutiendrait donc l'hypothèse que notre régime favoriserait la part de la digestion azotée effectuée dans le cæcum.

La valeur du CUDa de la cellulose brute (CB) au niveau cæcal (40,9 p. 100) est double de celle du CUDa obtenue par collecte. En l'absence de résultats complémentaires, nous ne pouvons expliquer cet écart important.

\section{TABleau 5}

Bilans digestifs totaux et par segment du tube digestif.

Total apparent digestibilities.

\begin{tabular}{l|r|r|r|r|c|c}
\hline \hline $\begin{array}{c}\text { CUDa \% } \\
\text { Apparent digestibility \% }\end{array}$ & $\begin{array}{c}\text { Pré- } \\
\text { cæcale }\end{array}$ & Cacale & $\begin{array}{c}\text { Post- } \\
\text { cæcale }\end{array}$ & Total & (1) & (2) \\
\hline Matière sèche - DM ....... & 14,1 & 32,2 & 7,6 & 53,9 & 53,1 & 54,5 \\
MAT (N $\times 6,25)$ - Crude protein & $-56,6$ & 36,6 & - & - & 56,8 & 60,5 \\
Cellulose brute - Crude fibre .. & - & 40,9 & - & - & 20,4 & 21,7 \\
\hline (1) Valeurs des CUDa, calculées à l'aide des concentrations en marqueur des fèces. \\
Apparent digestibility values calculated by means of the faecal concentration of markers. \\
(2) CUDa obtenus par collecte totale des fèces (voir tableau 1). \\
Apparent digestibility values obtained by total collection of faeces (see table 1). \\
\hline
\end{tabular}


Nous pouvons comparer les valeurs des CUDa obtenus par collecte totale des fèces et celles calculées à l'aide de la concentration en chrome des fèces (tabl. 5). Nous constatons alors que les écarts entre les 2 valeurs sont peu élevés, sauf dans le cas des matières azotées $(3,4 \mathrm{p}$. 100). Une récolte fécale supérieure à 3 jours permettrait probablement de réduire les écarts observés. Quoi qu'il en soit il semble possible de calculer un CUDa en évitant une récolte totale (quantitative) des fèces, si l'aliment contient un marqueur tel que le chrome mordancé.

\section{Discussion générale. Conclusion}

Les variations de concentration du marqueur en fonction du temps au niveau d'un même segment (fig. 1), expriment des variations de l'efficacité de la digestion ou des vitesses de transit différentielles; et ces variations semblent liées à la pratique de la cæcotrophie (fig. 3). Chez le lapin, le côlon proximal effectue un tri de particules (BJORNhaG, 1972) en période d'excrétion de crottes dures (entre $15 \mathrm{~h}$ et $3 \mathrm{~h}$ lors de nos essais). Les particules les plus fines (diamètre $<0,1 \mathrm{~mm}$ ) sont refoulées vers le cæcum, alors que les plus grosses (dont les fibres mordancées) constituent les crottes dures. Celles-ci ont donc une teneur en chrome (0,45 p. $100 \mathrm{MS})$ supérieure à celle des cæcotrophes (0,4 p. $100 \mathrm{MS}$; fig. 1 - Rectum).

Lors de l'ingestion des cacotrophes la teneur en chrome de l'estomac s'élève, puisque l'aliment granulé ne contient que 0,2 p. 100 de $\mathrm{Cr}_{2} \mathrm{O}_{3}$. Puis, parallèlement à l'évacuation des crottes molles, cette teneur s'abaisse.

Toutefois ceci ne peut expliquer que les teneurs en marqueur dans l'estomac, soient supérieures à celles de l'iléon. L'hypothèse suivante peut être avancée. Entre $9 \mathrm{~h}$ et $15 \mathrm{~h}$, nous trouvons dans l'estomac (fig. 4) deux fractions distinctes : l'aliment localisé dans l'antrum et les cæcotrophes dans le fundus. La fraction "aliment » $\left(0,2\right.$ p. 100 de $\left.\mathrm{Cr}_{2} \mathrm{O}_{3}\right)$ aurait un temps de séjour plus court que la phase "cæcotrophes » $\left(0,4\right.$ p. 100 de $\left.\mathrm{Cr}_{2} \mathrm{O}_{3}\right)$; ainsi les digesta quittant l'estomac auraient une concentration en marqueur inférieure à celle du contenu stomacal moyen. La rétention des cæcotrophes dans l'estomac expliquerait donc les concentrations élevées en marqueur à ce niveau.

L'activité de refoulement du côlon proximal, en période de formation de crottes dures, se traduit par un flux vers le cæcum de matière peu «fibreuse » et donc peu «marquée»; d'où la baisse de la teneur en $\mathrm{Cr}_{2} \mathrm{O}_{3}$ cæcal entre $15 \mathrm{~h}$ et $6 \mathrm{~h}$.

De même que Catala (1976), nous observons des variations journalières de la composition des digesta, en relation avec la pratique de la cacotrophie. Ceci montre donc que chez le lapin, il est nécessaire d'effectuer des prélèvements de contenus digestifs à plusieurs moments de la journée, si l'on désire obtenir des échantillons représentatifs.

L'estimation de la quantité moyenne de cæcotrophes produits souligne l'intérêt nutritionnel de la cæcotrophie, en particulier quant à l'apport azoté (bonne qualité des protéines d'origine bactérienne). La technique que nous employons est sans doute plus lourde que la pose d'un collier pour empêcher l'ingestion des crottes molles; mais elle évite le stress (baisse de la consommation, etc.), et elle permet de conserver les animaux dans une situation physiologique normale au cours de l'expérimentation. 
Notre marqueur semble correct si l'on s'en tient au calcul du bilan digestif de la matière sèche. Mais, si l'estimation au niveau stomacal, du flux d'azote et de cellulose brute semble correcte, il n'en est pas de même au niveau iléal et cæcal : sous-estimation dans le cas de l'azote. Wolter et al. (1980) pensent que les abondantes sécrétions digestives endogènes expliquent un CUDa de l'azote négatif au niveau de l'iléon. Cette hypothèse ne peut seule, expliquer qu'une part des valeurs obtenues. D'autres éléments doivent augmenter le flux iléal d'azote : tels que, la technique de prélèvement, ou l'abattage par dislocation cervicale qui pourrait entraîner une desquamation brutale de l'épithélium... Dans ce domaine, la fistulation iléale du lapin pourrait peut-être apporter des réponses plus précises.

De plus, il existe sans doute un transit différentiel entre les composants de la ration. Le marquage de l'un de ces composés semble donc insuffisant, d'après ces premiers résultats. Une étude plus approfondie est nécessaire : expérience sur un nombre plus important d'animaux, allongement de la période de récolte des fèces...

Cette méthodologie qui s'est montrée intéressante pour l'étude des flux de matière sèche doit aussi être testée précisément par le calcul de bilans digestifs des constituants pariétaux. Nous pourrons alors mieux apprécier l'efficacité de la flore cæcocolique du lapin, dans la digestion des constituants de la paroi végétale.

Reçu en août 1985.

Accepté en novembre 1985.

\begin{abstract}
Summary
Digestion of a high fibre diet in growing rabbits : methodological study for determining partial apparent digestibility
\end{abstract}

A new methodology was used in growing rabbits with the aim of estimating digestion in the different segments of the digestive tract. Twenty-seven 8-week old white New Zealand rabbits received a dict rich in cell wall carbohydrates $(25$ p. 100 crude fibre $)$ and lignin (11 p. 100) admixed with chromium oxide used as a marker. Digesta were collected after slaughter every $3 \mathrm{~h}$ for a period of $24 \mathrm{~h}$ from the stomach, terminal ileum, caecum and rectum. The apparent digestibility coefficients (CUDa), calculated by total collection of faeces, were low for dry matter, energy and crude protein (54-60 p. 100, table 1). Hemicelluloses (VAN SoEST) were better digested $(\mathrm{CUDa}=40 \mathrm{p} .100)$ than cellulose $(\mathrm{CUDa}=30 \mathrm{p} .100)$ while 16 p. 100 lignin seemed to be digested.

Combining the slaughter technique with the use of food markers allowed to determine the apparent digestibility and mean retention times at the different collection sites as well as the amount of caecotrophes. Thus, the apparent digestibility of dry matter was 14 p. 100 before the caecum versus $32 \mathrm{p} .100$ in the caecum. However, this method was not as suitable for measuring nitrogen digestion (e.g. negative CUDa in the ileum). The hypothesis of a differential passage of dietary components was put forward. The average apparent retention times (table 4) were 3-4 $\mathrm{h}$ for the stomach and 4-6 $\mathrm{h}$ for the caecum. These values are lower than those of the literature. The daily amount of caecotrophes reached 15 p. 100 of the total dry matter intake and 23 p. 100 of the total crude protein intake. trophy.

Key words : Digestibility, food passage, cell wall constituants, growing rabbit, caeco- 


\section{Références bibliographiques}

AkıN D.E., 1982. Microbial breakdown of feed in the digestive tract. «Nutritional limits to animal production from pastures ». Ed. : J.B. Hacker (C.A.B.) symp. 24-28 août 1981, Sainte-Lucie, Australie.

BJORNHAG G., 1972. Separation and delay of contents in the rabbit colon. Swedish J. agric. Res., 2, 125-136.

Catala J., 1976. Etudes sur les répartitions hydrique, pondérale et azotée dans le matériel digestion chez le lapin, en relation avec la dualité de l'élimination fécale. $1^{\mathrm{er}}$ congrès int. cunicole. Dijon, communication $\mathbf{n}^{\circ} 58$.

Colin M., Lebas F., 1976. Méthode d'étude de la digestibilité des aliments : II. Périodicité des collectes. Sci. Tech. Anim. Labo, 1, 129-133.

Demaux G., Guemon L., Gallouin F., 1980. Evolution temporelle des relations entre quelques. paramètres biochimiques et histologiques à différents niveaux du tube digestif chez le lapin. Assoc. Fr. Nutr. J. Digestion-absorption. 20-21 nov. Reprod. Nutr. Develop., 1981, 21 (5 B), 868.

Ellis W.C., Huston J.E., $1968 .{ }^{144} \mathrm{Ce}-{ }^{144} \mathrm{Pr}$ as a particulate digesta flow marker in ruminants. J. Nutr., 95, 67-78.

FAICHNEY G.J., 1980. Measurement in sheep of the quantity and composition of rumen digesta and of the fractionnal outflow rates of digesta constituents. Aust. J. Agric. Res., 31, 1129-1137.

Gidenne T., Lebas F., 1984. Evolution circadienne du contenu digestif chez le lapin en croissance. Relation avec la cæcotrophie. 3" Congrès Mondial de Cuniculture. Rome, vol. 2, 494-501.

HoRnICKe H., 1980. Utilization of caecal digesta by caecotrophy (soft faeces ingestion) in the rabbit. 31. Jahrestagung der Europäischen Vereinigung für Tierzucht. 1-4 september 1980 .

Kametaka M., 1967. Studies on the digestion in the rabbit. Part XI : some physicochemical properties of digesta in the small intestinal tract of the rabbit fed on a pellet diet. Agr. Biol. Chem., 31, 616-624.

Kotв R.A., Luckey T.D., 1972. Markers in nutrition. Nutr. Abst. Rev., 42, 813-845.

LAPLACE J.P., 1978. Le transit digestif chez les monogastriques. III. Comportement (prise de nourriture, cæcotrophie), motricité et transit digestif et pathogénie des diarrhées chez le lapin. Ann. Zootech., 27, 225-265.

Le Bars H., Guemon L., Demaux G., 1971. Production d'acides gras volatils dans le cæcum du lapin. Ann. Biol, anim. Biochim. Biophys., 11, 301-302 (Abstr.).

LEBAS F., 1975. Influence de la teneur en énergie de l'aliment sur les performances de croissance du lapin. Ann. Zootech., 24, 281-288.

LeBas F., 1979. La nutrition du lapin : mouvement des digesta et transit. Cuniculture, 6, 67-68.

Lebas F., Laplace J.P., 1977. Le transit digestif chez le lapin : VIII. Influence de la source de cellulose. Ann. Zootech., 26, 575-584.

Lebas F., Laplace J.P., Droumeno P., 1982. Effets de la teneur en énergie de l'aliment chez le lapin. Variations en fonction de l'âge des animaux et de la séquence des régimes alimentaires. Ann. Zootech., 31, 233-256.

Leng E., Hornicke H., 1975. Tagesrhythmische Unterschiede in der Zusammensetzung des Blinddarminhalts von Kaninchen. Z. Versuchstierk., 17, 285-299.

Mathieson J., 1970. Automated estimation of chromicoxyde. Proc. Nutr. Soc., 29, 30 A.

Minson D.J., 1966. The apparent retention of food in the reticulorumen at two levels of feeding by means of an hourly feeding technique. Br. J. Nutr., 20, 767-773.

RANDAll K.E., 1977. Digestion of dietary fiber and energy in the adult New-Zealand white rabbit. Thesis, Aberdeen University, Uk, 1976, 26 pp. 
Susmel P., Lanarı D., 1976. Changes in volatile fatty acid level in rabbit caecum. $f^{\text {er }}$ Congrès int. cunicole de Dijon, communication $\mathrm{n}^{\prime \prime} 32$.

UDEN P., 1978. Comparative studies on rate of passage, particle size and rate of digestion in ruminants, equines, rabbits and man. Thesis, Ph. D. Cornell University.

Uden P., Colucci E.P., Van Soest P.J., 1980. Investigation of chromium, cerium and cobalt as markers in digesta. Rate of passage studies. J. Sci. Fd. Agric., 31, 625-632.

VAN Soest P.J., Wine R.H., 1967. Use of detergents in the analysis of fibrous feeds. IV. Determination of plant cell-well constituent. J. Ass. Off. Agric. Chem., 50, 50-55.

Yoshida T., Kandatsu M., 1967. Studies on caecum digestion. VII. On the operation for caecum fistula in rabbit and excretion of orally administrated $\mathrm{Cr}_{2} \mathrm{O}_{*}$ to hard and soft feces. Jap. J. Zootech. Sci., 38, 358-363.

Yoshihara I., Kandatsu M., 1960. Studies on caecum digestion. IV. On the movement of caecal contents in the rabbit. Bul. Agr. Chem. Soc. Japan, 24, 543-547. Cité par LAPLACE (1978).

Wolter R., Nouwakpo F., Durix A., 1980. Etude comparative de la digestion d'un aliment complet chez le poney et le lapin. Reprod. Nutr. Dévelop., 20, 1723-1730. 